\title{
A six-microRNA panel in plasma was identified as a potential biomarker for lung adenocarcinoma diagnosis
}

\author{
Xin Zhou ${ }^{1, *}$, Wei Wen ${ }^{2, *}$, Xia Shan ${ }^{3, *}$, Wei Zhu ${ }^{1}$, Jing $\mathrm{Xu}^{1}{ }^{1}$, Renhua Guo ${ }^{1}$, Wenfang \\ Cheng ${ }^{4}$, Fang Wang ${ }^{5}$, Lian-Wen $\mathbf{Q i}^{6}$, Yan Chen ${ }^{7}$, Zebo Huang ${ }^{1}$, Tongshan Wang ${ }^{1}$, \\ Danxia Zhu ${ }^{8}$, Ping Liu ${ }^{1,9}$, Yongqian Shu ${ }^{1,9}$ \\ ${ }^{1}$ Department of Oncology, First Affiliated Hospital of Nanjing Medical University, Nanjing 210029, PR China \\ ${ }^{2}$ Department of Thoracic Surgery, First Affiliated Hospital of Nanjing Medical University, Nanjing 210029, PR China \\ ${ }^{3}$ Department of Respiration, The Affiliated Jiangning Hospital of Nanjing Medical University, Nanjing 210000, PR China \\ ${ }^{4}$ Department of Gastroenterology, First Affiliated Hospital of Nanjing Medical University, Nanjing 210029, PR China \\ ${ }^{5}$ Department of Cardiology, First Affiliated Hospital of Nanjing Medical University,Nanjing 210029, PR China \\ ${ }^{6}$ State Key Laboratory of Natural Medicines and Department of Pharmacognosy, China Pharmaceutical University, Nanjing, \\ 210009, China \\ ${ }^{7}$ Department of Emergency, First Affiliated Hospital of Nanjing Medical University, Nanjing 210029, PR China \\ ${ }^{8}$ Department of Oncology, The Third Affiliated Hospital of Soochow University, Changzhou 213003, China \\ ${ }^{9}$ Cancer Center of Nanjing Medical University, Nanjing 210029, China \\ *These authors contributed equally to this work \\ Correspondence to: Wei Zhu, email: zhuwei@njmu.edu.cn \\ Ping Liu, email: liupinga28@163.com \\ Yongqian Shu, email: shuyongqian@njmu.edu.cn
}

Keywords: plasma, miRNA, lung adenocarcinoma, diagnosis, exosomes

Received: July 25, 2016

Accepted: December 13, 2016

Published: December 27, 2016

\section{ABSTRACT}

Differently expressed microRNAs (miRNAs) in the plasma of lung adenocarcinoma (LA) patients might serve as biomarkers for LA detection. MiRNA expression profiling was performed using Exiqon panels followed by the verification ( 30 LA VS. 10 healthy controls (HCs)) with quantitative reverse transcription polymerase chain reaction (qRT-PCR) in the screening phase. Identified miRNAs were confirmed through training (42 LA VS. 32 HCs) and testing stages ( 66 LA VS. 62 HCs) by using qRT-PCR based absolute quantification methods. A total of six up-regulated plasma miRNAs (miR-19b3p, miR-21-5p, miR-221-3p, miR-409-3p, miR-425-5p and miR-584-5p) were identified. The six-miRNA panel could discriminate LA patients from HCs with areas under the receiver operating characteristic curve of $0.72,0.74$ and 0.84 for the training, testing and the external validation stage ( 33 LA VS. $30 \mathrm{HCs}$ ), respectively. All the miRNAs identified except miR-584-5p were significantly up-regulated in LA tissues. MiR-19-3p, miR-21-5p, miR-409-3p and miR-425-5p showed high expression in arterial plasma with borderline significance. Additionally, miR-19-3p, miR-21-5p and miR-221-3p were significantly up-regulated in exosomes extracted from LA peripheral plasma samples. In conclusion, we identified a six-miRNA panel in peripheral plasma which might give assistance to the detection of LA at least for Asian population to a certain extent.

\section{INTRODUCTION}

Lung cancer is one of the most fatal malignancies and the leading cause of cancer-related death worldwide [1]. Occupying more than $80 \%$ of lung cancer, nonsmall cell lung cancer (NSCLC) includes squamous cell carcinoma (SCC), large cell carcinoma and adenocarcinoma (LA) $[2,3]$. Surgical resection remains to be the most effective treatment for NSCLC at present. However, due to lack of apparent symptoms, many patients are diagnosed with locally advanced and advanced stages and do not have a choice of surgery which leads 
to a low 5-year overall survival rate (less than $15 \%$ ) for NSCLC patients. Despite increased understanding of the molecular and clinical characteristics of NSCLC as well as recent advances in screening and treatment strategies [4-7], the prognosis of NSCLC is still poor. Computed tomography (CT) especially low-dose CT is currently used as the screening method for NSCLC. But potential overdiagnosis and harmful effects induced by radiation limit their use in the clinical [8]. Existing protein biomarkers such as carcinoembryonic antigen (CEA) and CYFRA21-1 did not show sufficient sensitivity and specificity $[9,10]$. In view of this, novel and reliable biomarkers to detect NSCLC for early intervention with the potential to reduce mortality are urgently needed.

MicroRNAs (miRNAs) are short (typically 1825 nucleotides), single-stranded and highly conserved non-coding RNAs which could negatively regulate gene expression at post-transcriptional level by binding the $3^{\prime}$-untranslated region of target mRNAs, resulting in either mRNA degradation or translational repression $[11,12]$. Passively leaked or actively transported from cells, circulating miRNAs could be stably detected in blood and have been used as biomarkers for diagnosis, prognosis or monitoring curative effect in various cancers including NSCLC [13]. Most previous studies focused on the differential expression levels of miRNAs in patients with NSCLC, however, the results were not consistent due to diverse research methods and tested populations [14]. Different subtypes of NSCLC showed different molecular alterations, biological behaviors and might also own different miRNA profilings. Recent clinical trials have shown that sub-grouping NSCLC into subtypes could achieve maximum benefit while minimising toxicity for patients $[15,16]$. Comprehensive molecular profiling of LA (the largest subgroup of NSCLC) revealed by The Cancer Genome Atlas (TCGA) project could also establish a foundation for classification of NSCLC [17]. Thus, there is an urgent need in considering subtypes when aiming to identify biomarkers. In addition, differences of preanalytical and analytical variables could impact the accuracy of detecting circulating miRNAs [18]. However, absolute quantification procedure based on the spiked-in normalization method seems to be the optimal way to normalize miRNA between body fluid samples [19-21].

In the present study, we focused on the specific subtype of NSCLC (LA) and conducted a four-phase study to identify potential miRNAs for detecting LA by evaluating the absolute concentration of plasma miRNAs based on quantitative reverse transcription polymerase chain reaction (qRT-PCR). In addition, the identified miRNAs were confirmed in tissue samples and compared in arterial and peripheral plasma samples. Exosomal miRNAs were also investigated to assess the potential form of the identified miRNAs in peripheral plasma which might aid detection of LA.

\section{RESULTS}

\section{Characteristics of subjects}

A total of 265 subjects, including 141 LA patients and 124 healthy controls (HCs), were included in our study. The flow chart of the experiment (Figure 1) showed that our study was divided into three stages after the screening phase: the training stage, the testing stage, and the external validation stage. Each pooled sample analyzed in the screening phase was randomly selected from the subjects, and per 10 samples were pooled as 1 pool sample. As shown in Table 1, there was no significant difference in the distribution of age or gender between LA patients and $\mathrm{HCs}$ in any stage ( $p$-values $>0.05)$.

\section{MiRNA profiling from the screening phase}

To identify differentially expressed miRNAs in peripheral plasma of LA patients, we initially screened 168 miRNAs by the Exiqon miRCURY-Ready-to-UsePCR-Human-panel-I + II-V1.M on the qRT-PCR platform in $3 \mathrm{LA}$ and $1 \mathrm{HC}$ pooled samples. Only the miRNAs with cycle threshold $(\mathrm{Ct})$ value $<37$ and 5 lower than negative control in the panel were eligible for further analysis. A total of 39 miRNAs (36 up-regulated miRNAs and 3 down-regulated miRNAs; Supplementary Table S1 online) showed more than two-fold altered expression in all 3 pooled LA samples compared to the HC pool sample and were selected as candidate miRNAs. After that, to verify the reproducibility of the results from arrays and control the false discovery rate, the 39 miRNAs were confirmed by qRT-PCR in the same 40 participants. Fourteen miRNAs (Supplementary Table S1 online) including 13 up-regulated and 1 down-regulated miRNAs with mean fold change (FC) $>1.5$ or $<0.66$ and $P$ value $<0.05$ were identified and chosen to further validation stage outlined below.

\section{Validation of the miRNAs in peripheral plasma by qRT-PCR}

The expression levels of the 14 miRNAs identified through the screening phase were evaluated in a larger sample set including $42 \mathrm{LA}$ patients and $32 \mathrm{HCs}$ in the training stage. Six miRNAs (miR-19b-3p, miR-21-5p, miR-221-3p, miR-409-3p, miR-425-5p and miR-584-5p) were found to be significantly up-regulated in plasma from LA patients (Table 2; the other 8 miRNAs were shown in the Supplementary Table S2). Next, the six miRNAs were subjected to validation in the testing stage with 66 LA patients and $62 \mathrm{HCs}$. And the differential expression patterns of the six miRNAs between LA patients and HCs were concordant between the training and the testing stage (Table 2). In addition, all the six miRNAs had significantly higher expression levels in peripheral plasma of LA 
Table 1: Characteristics of 141 LA patients and 124 healthy controls included in the study

\begin{tabular}{|c|c|c|c|c|c|c|c|c|}
\hline \multirow[b]{2}{*}{ Variables } & \multicolumn{2}{|c|}{$\begin{array}{l}\text { Screening phase } \\
\quad(n=40)\end{array}$} & \multicolumn{2}{|c|}{$\begin{array}{l}\text { Training cohort } \\
\quad(n=74)\end{array}$} & \multicolumn{2}{|c|}{$\begin{array}{l}\text { Testing cohort } \\
\quad(n=128)\end{array}$} & \multicolumn{2}{|c|}{$\begin{array}{l}\text { External validation cohort } \\
\qquad(n=63)\end{array}$} \\
\hline & Cases (\%) & Controls (\%) & Cases (\%) & Controls (\%) & Cases (\%) & Controls (\%) & Cases (\%) & Controls (\%) \\
\hline Number & 30 & 10 & 42 & 32 & 66 & 62 & 33 & 30 \\
\hline \multicolumn{9}{|l|}{ Gender } \\
\hline Male & $14(46.7)$ & $4(40)$ & $16(38.1)$ & $15(46.9)$ & $30(45.4)$ & $28(45.2)$ & $15(45.5)$ & $14(46.7)$ \\
\hline Female & $16(53.3)$ & $6(60)$ & $26(61.9)$ & $17(53.1)$ & $36(54.6)$ & $34(54.8)$ & $18(54.5)$ & $16(53.3)$ \\
\hline \multicolumn{9}{|l|}{ Age } \\
\hline$<60$ & $12(40)$ & $5(50)$ & $24(57.1)$ & $20(62.5)$ & $28(42.4)$ & $30(48.4)$ & $16(48.5)$ & $13(43.3)$ \\
\hline$\geq 60$ & $18(60)$ & $5(50)$ & $18(42.9)$ & $12(37.5)$ & $38(57.6)$ & $32(51.6)$ & $17(51.5)$ & $17(56.7)$ \\
\hline \multicolumn{9}{|l|}{ Smoking } \\
\hline Smoker & $2(6.7)$ & & $2(4.8)$ & & $5(7.6)$ & & $3(9.1)$ & \\
\hline Non-smoker & $21(70)$ & & $37(88.1)$ & & $57(86.4)$ & & $28(84.9)$ & \\
\hline NA & $7(23.3)$ & & $3(7.1)$ & & $4(6)$ & & $2(6)$ & \\
\hline \multicolumn{9}{|l|}{ TNM stage } \\
\hline I & $9(30)$ & & $14(33.3)$ & & $18(27.3)$ & & $9(27.3)$ & \\
\hline II & $5(16.7)$ & & $9(21.4)$ & & $15(22.7)$ & & $8(24.2)$ & \\
\hline III & $5(16.7)$ & & $10(23.9)$ & & $19(28.7)$ & & $10(30.3)$ & \\
\hline IV & $11(36.6)$ & & $9(21.4)$ & & $14(21.3)$ & & $6(18.2)$ & \\
\hline \multicolumn{9}{|c|}{ EGFR mutation } \\
\hline Yes & $5(16.7)$ & & $8(19.1)$ & & $14(21.2)$ & & $4(12.1)$ & \\
\hline No & $6(20)$ & & $7(16.7)$ & & $8(12.1)$ & & $5(15.2)$ & \\
\hline NA & $19(63.3)$ & & $27(64.2)$ & & 44 (66.7) & & $24(72.7)$ & \\
\hline
\end{tabular}

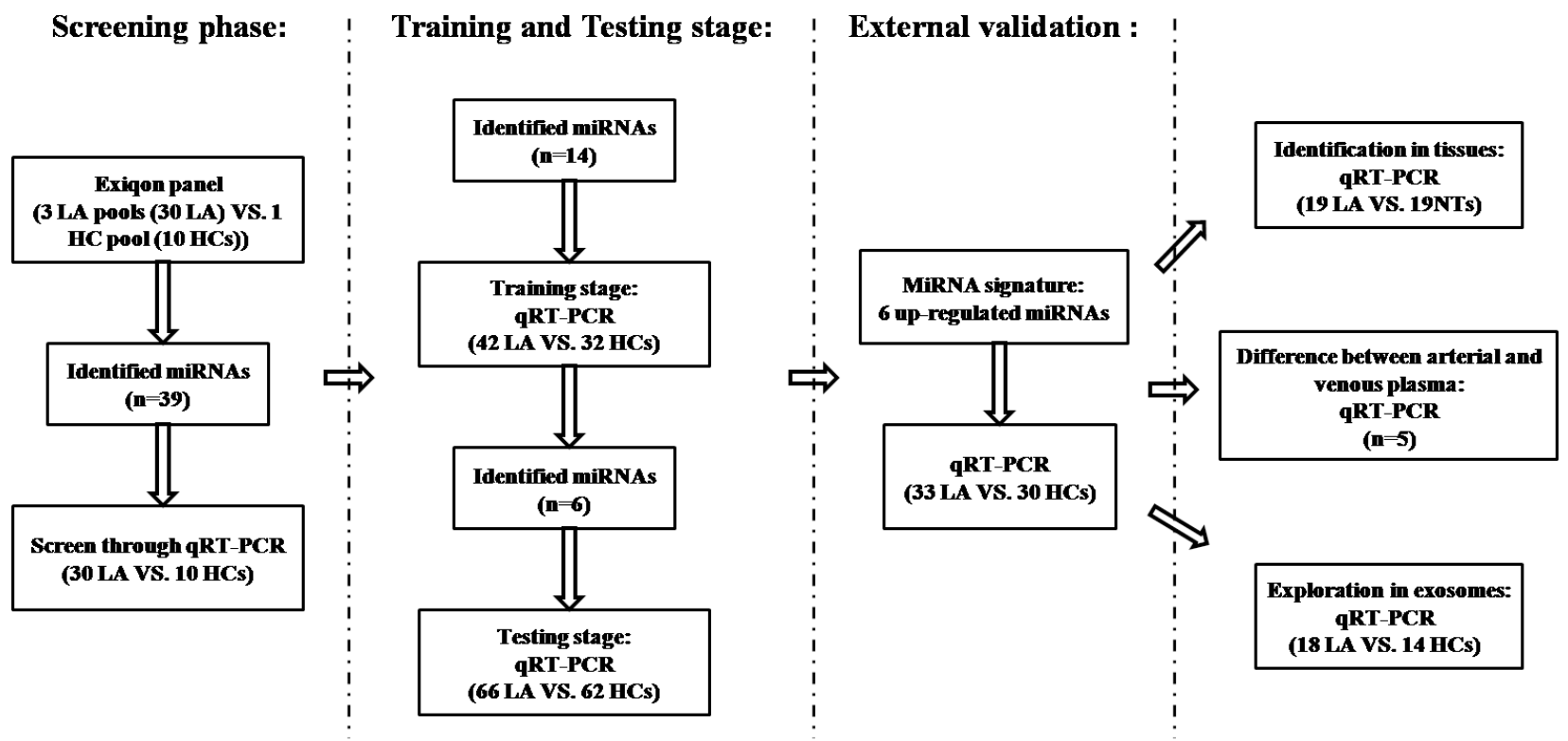

Figure 1: The flow chart of the experiment design. LA: lung adenocarcinoma; HC: healthy control; NT: normal tissue. 
Table 2: Expression levels of the six miRNAs in the peripheral plasma in the training and testing stage (presented as mean $\pm \mathrm{SD} ; \mathrm{fmol} / \mathrm{L}$ )

\begin{tabular}{lcccccccccc}
\hline \multicolumn{1}{c}{ miRNA } & \multicolumn{3}{c}{ Training stage } & \multicolumn{3}{c}{ Testing stage } & \multicolumn{2}{c}{ Combined } \\
\hline & Cases & Controls & FC & $\boldsymbol{P}$ value & Cases & Controls & FC & $P$ value & FC & $\boldsymbol{P}$ value \\
\hline miR-19b-3p & $592 \pm 458$ & $331 \pm 222$ & 1.79 & 0.015 & $517 \pm 447$ & $313 \pm 280$ & 1.65 & 0.022 & 1.71 & $<0.001$ \\
miR-21-5p & $1338 \pm 1567$ & $632 \pm 709$ & 2.12 & 0.005 & $1103 \pm 990$ & $570 \pm 546$ & 1.93 & $<0.001$ & 2.02 & $<0.001$ \\
miR-221-3p & $127 \pm 136$ & $51 \pm 56$ & 2.49 & 0.003 & $132 \pm 194$ & $59 \pm 81$ & 2.23 & 0.001 & 2.31 & $<0.001$ \\
miR-409-3p & $14 \pm 17$ & $3.3 \pm 5.5$ & 4.26 & 0.005 & $12.3 \pm 16$ & $2.8 \pm 2.4$ & 4.45 & 0.033 & 4.39 & $<0.001$ \\
miR-425-5p & $262 \pm 195$ & $152 \pm 69$ & 1.72 & 0.017 & $201 \pm 166$ & $128 \pm 64$ & 1.57 & 0.015 & 1.64 & $<0.001$ \\
miR-584-5p & $330 \pm 458$ & $161 \pm 197$ & 2.05 & 0.007 & $353 \pm 425$ & $145 \pm 164$ & 2.43 & $<0.001$ & 2.28 & $<0.001$ \\
\hline
\end{tabular}

FC: fold change.

patients as compared with HCs when the results of the two stages were combined (Table 2; Figure 2).

\section{Diagnostic value of miRNAs in peripheral plasma}

To evaluate the performance of the six miRNAs in discriminating LA patients from HCs, the optimal cutoff values for miR-19b-3p, miR-21-5p, miR-221-3p, miR409-3p, miR-425-5p and miR-584-5p were determined according to ROC curves for each miRNA in the combined cohorts from the training and testing stage. AUCs for miR19b-3p, miR-21-5p, miR-221-3p, miR-409-3p, miR-425$5 \mathrm{p}$ and miR-584-5p were 0.62 (95\% confidence interval (CI): $0.54-0.7), 0.69$ (95\% CI: $0.62-0.77), 0.68(95 \%$ CI: $0.61-0.76), 0.61$ (95\% CI: $0.53-0.69), 0.66$ (95\% CI:
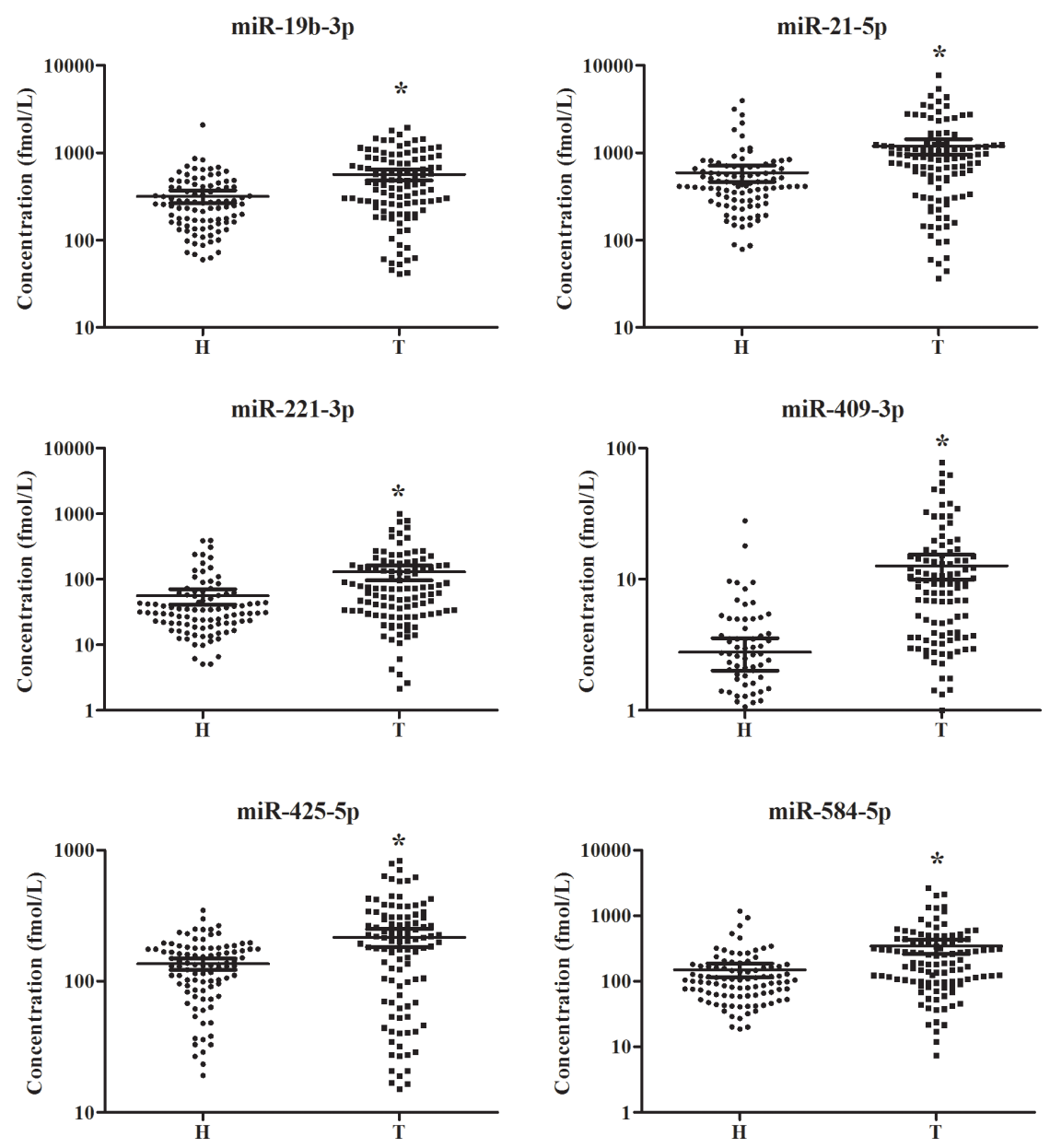

Figure 2: Expression levels of the six miRNAs in the peripheral plasma of $108 \mathrm{LA}$ patients and 94 controls (in the training and testing stages). $\mathrm{H}$ : healthy controls; T: tumor. Horizontal line: mean with $95 \% \mathrm{CI}$. ${ }^{*} P$-value $<0.05$. 
Table 3: Expression levels of the six miRNAs in the peripheral exosomes of $18 \mathrm{LA}$ patients and 14 HCs (presented as mean $\pm \mathrm{SD} ; 2^{-\Delta \Delta C t}$ )

\begin{tabular}{lcccc}
\hline \multicolumn{1}{c}{ miRNA } & Cases & Controls & FC & P value \\
\hline miR-19b-3p & $0.79 \pm 0.85$ & $1.05 \pm 0.32$ & 1.3 & 0.02 \\
miR-21-5p & $0.49 \pm 0.73$ & $1.2 \pm 0.82$ & 2.46 & $<0.001$ \\
miR-221-3p & $0.39 \pm 0.24$ & $1.17 \pm 0.65$ & 3.02 & $<0.001$ \\
miR-409-3p & $0.81 \pm 1.44$ & $1.29 \pm 1.61$ & 1.59 & 0.111 \\
miR-425-5p & $0.98 \pm 1.05$ & $1.37 \pm 1.25$ & 1.39 & 0.129 \\
miR-584-5p & $0.62 \pm 0.69$ & $1.06 \pm 0.62$ & 1.69 & 0.063 \\
\hline
\end{tabular}

FC: fold change.

$0.58-0.74)$ and 0.69 (95\% CI: $0.62-0.76)$, respectively (Supplementary Figure S1 online). To evaluate the diagnostic utility of the six-miRNA panel, the risk score function (RSF) of the six miRNAs for each subject was calculated. As shown in Figure 3A, the six-miRNA panel had a greater ability in distinguishing different groups than one particular miRNA in the combined cohorts with AUC of 0.73 (95\% CI: $0.66-0.8$; sensitivity $=68 \%$ and specifity $=70 \%$ ). By using the same cutoff values, the diagnostic value of the six-miRNA panel was also assessed in the training and testing stage separately. The AUC of the panel was 0.72 (95\% CI: $0.6-0.83$; sensitivity $=69 \%$ and specifity $=66 \%$ ) and 0.74 (95\% CI: $0.65-0.82$; sensitivity $=67 \%$ and specifity $=71 \%$ ) for the training and the testing stage, respectively (Figure 3B and 3C).

To further confirm the diagnostic capacity of the six-miRNA panel for diagnosing LA, an additional cohort including $33 \mathrm{LA}$ patients and $30 \mathrm{HCs}$ was explored. Consistent with the results in the training and the testing stage, all six miRNAs showed significantly high expression level in plasma of LA patients (Supplementary Table S3 online). As shown in Figure 3D, the six-miRNA panel could accurately discriminate LA patients from HCs with AUC of 0.84 (95\% CI: 0.75-0.94; sensitivity $=73 \%$ and specifity $=80 \%$ ).

To explore the relationship of the six-miRNA panel with clinical parameters, we analyzed data of all 141 LA patients. No miRNA or RSF was significantly related with gender, age, smoking status or tumor stage ( $p$-values $>$ $0.05)$. Epidermal growth factor receptor (EGFR) mutation status was examined in 46 patients in our study as part of routine pathology to guide decision-making regarding adjuvant therapy in the clinical (20 cases with wild type, 12 with DelE746-A750 mutation and 14 with L858R mutation). No significant difference of the six-miRNA panel was found in 20 patients with wild type and 26 cases with EGFR mutation. However, we found that plasma miR-19b-3p and miR-425-5p were significantly up-regulated in 12 patients with DelE746-A750 mutation compared to those with wild type (Figure 4).

\section{Evaluation of miRNAs in tissue samples}

Next, we examined the expression levels of the six miRNAs in an additional 19 pairs of tissue samples to evaluate the relationship of the identified miRNAs in peripheral plasma and tissues of LA patients. As shown in Figure 5, all the six miRNAs except miR-584-5p had higher expression in tumor samples than in normal tissues.

\section{Comparison of miRNAs in peripheral and arterial plasma}

As blood flows from arterial to venous circulation, we assessed expression levels of the miRNAs in 5 arterial plasma samples and compared those in the matched peripheral plasma samples from the same individuals. Among the six miRNAs, miR-19-3p, miR-21-5p, miR221-3p, miR-409-3p and miR-425-5p showed higher expression levels in arterial plasma in more than a half of the subjects. However, due to the relatively small sample size, the results were not statistically significant (Figure 6).

\section{Identification of miRNAs in peripheral plasma exosomes}

In addition, the expression levels of miRNAs in exosomes from $18 \mathrm{LA}$ and $14 \mathrm{HC}$ peripheral plasma samples were explored to assess the potential form of the identified miRNAs in peripheral plasma. All the six miRNAs were up-regulated but only miR-19-3p, miR-21$5 \mathrm{p}$ and $\mathrm{miR}-221-3 \mathrm{p}$ were with statistical significance in exosomes from LA plasma samples (Table 3).

\section{DISCUSSION}

Majority of previous studies investigated the diagnostic performance of circulating miRNAs in NSCLC which comprised a range of subgroups with molecular and histological heterogeneity [15]. In the present study, we focused on LA and designed a four-phase study to identify 
peripheral plasma miRNAs which might have potential in detecting the disease. In the screening phase, differential expression profiling of plasma miRNAs were initially analyzed in $3 \mathrm{LA}$ and $1 \mathrm{HC}$ pooled samples with Exiqon miRNA qPCR panels which appeared to show better sensitivity and linearity than TaqMan platform while less abundant miRNAs were measured [22]. To control the false positive rate, miRNAs identified from Exiqon panels were confirmed in the same 40 plasma samples by qRTPCR. In the following validation stages, the expression levels of plasma miRNAs were assessed by the method of absolute concentration analysis which might be the optimal way to normalize miRNA in body fluid samples. Through the training and the testing stage, a miRNA panel including six up-regulated peripheral plasma miRNAs (miR-19b-3p, miR-21-5p, miR-221-3p, miR-409-3p, miR425-5p and miR-584-5p) was identified and could be used as a biomarker in diagnosis of LA. The external validation stage further verified the reliability of the diagnostic value of the six-miRNA panel. The six miRNAs were also explored in LA tissues. However, miR-19b-3p, miR-21-5p, miR-221-3p, miR-409-3p and miR-425-5p but not miR584-5p showed consistently high expression in LA tissues.
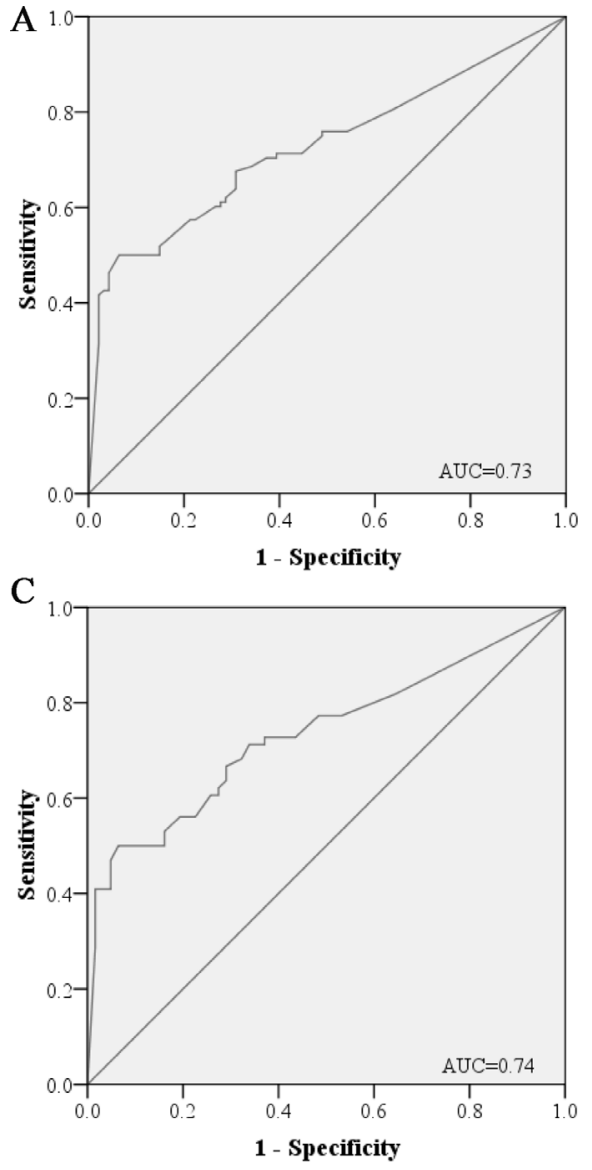

Among the six miRNAs identified in our study, miR-19b-3p was one of the key oncogenic components of the miR-17-92 cluster. High miR-19b-3p expression in NSCLC tissues was related with higher TNM stage, lymph node metastasis and poorer survival. Enforced expression of miR-19b-3p could trigger epithelial-mesenchymal transition (EMT), enhance migration and invasion of lung cancer cells [23]. In addition, miR-19b-3p was also reported to be up-regulated in serum and served as an unfavorable predictor in prognosis of NSCLC [24]. As a well known onco-miRNA, miR-21-5p was studied thoroughly in various cancers including lung cancer $[25,26]$. High expression of miR-21-5p was found in tissue samples, blood samples and sputum samples and could be used as a predictor in diagnosis, prognosis and monitoring therapy effect for patients with NSCLC [27-32]. Recently, TCGA project focused on comprehensive, multiplatform analysis of LA, with attention towards pathobiology and clinically actionable events which might be beneficial to further investigations of lung cancer [17]. They also found miR-21-5p was one of the 32 most discriminatory miRNAs between the matched LA/adjacent normal samples These results were consistent
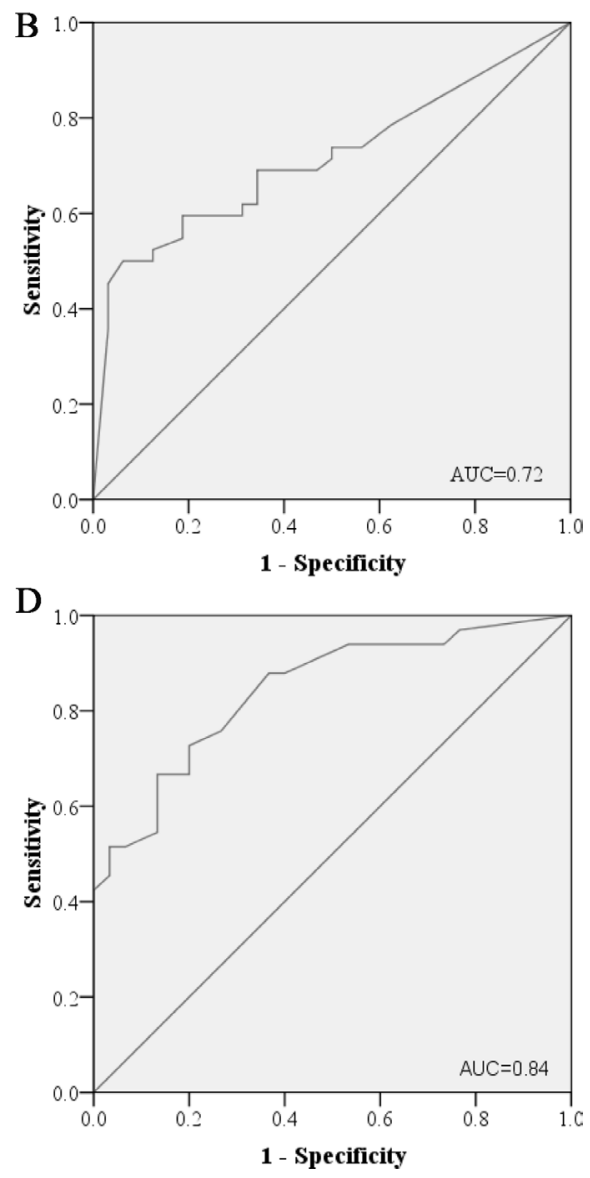

Figure 3: Receiver-operating characteristic (ROC) curves for the six-miRNA panel to discriminate LA patients from healthy controls. (A) the combined two cohorts of training and testing stages (108 LA VS. 94 HCs); (B) training stage (42 LA VS. 32 HCs); (C) testing stage (66 LA VS. 62 HCs); (D) external validation stage (33 LA VS. 30 HCs). LA: lung adenocarcinoma; HC: healthy control. AUC: areas under the curve. 
with our findings. As for miR-221-3p, controversial roles were found in the development and progression of lung cancer [33-35]. Heegaard et al. demonstrated that miR-221-3p was down-regulated in the serum but not in plasma sample of NSCLC patients [36]. But in our study, we found miR-221-3p was consistently up-regulated in plasma and tissues from LA patients. Thus, the conflicting function of miR-221-3p is needed to be further investigated. Though not studied in LA, miR-425-5p was found to be up-regulated and closely related to tumor stages and sizes in SCC tissues. It was also suggested that miR-425-5p might be a driver for tumor formation, growth, and progression to higher staging in SCC [37]. It was in accordance with our finding of miR-425-5p in LA. The other two miRNAs (miR-409-3p and miR-584-5p) were not explored in lung cancer and had distinct roles in different types of cancer [38-41]. In our study, miR-409$3 p$ was up-regulated in both plasma and tissue samples from LA patients and might play an important role in LA. For miR-584-5p, we found that it was up-regulated in LA plasma but did not show differential expression level between LA and normal tissues. According to the theory that circulating miRNAs are originated from cells, we might assume that up-regulated plasma miR-584-5p might be a passenger but not a driver factor for tumorgenesis in LA. Future research of these miRNAs in LA formation and development is warranted.

The diagnostic capacity of the miRNAs identified in our study was also explored in some other cancers. Down-regulation of miR-19b-3p was found in plasma of gastric cancer and could distinguish normal population from patients with different TNM stages and grades [42]. Circulating miR-21 was also assessed to be a diagnostic biomarker in various cancers such as hepatocelluar carcinoma, gastric cancer and so on $[43,44]$. Up-regulation of miR-221-3p in the circulation could also aid in the detection of gastric cancer, colorectal cancer, malignant melanoma, larynx cancer and pancreatic cancer [45-49]. To better take advantage of these miRNAs as biomarkers for specific cancer, future study is necessary.

As a member of the ErbB family of transmembrane receptor tyrosine kinases, EGFR is involved in signal transduction pathways that regulate apoptosis and proliferation [50]. NSCLC patients with activating mutations in the EGFR gene could benefit from the treatment with EGFR tyrosine kinase inhibitors (EGFRTKI). Thus, EGFR mutation status of NSCLC patients is determined in the clinical to guide decision-making
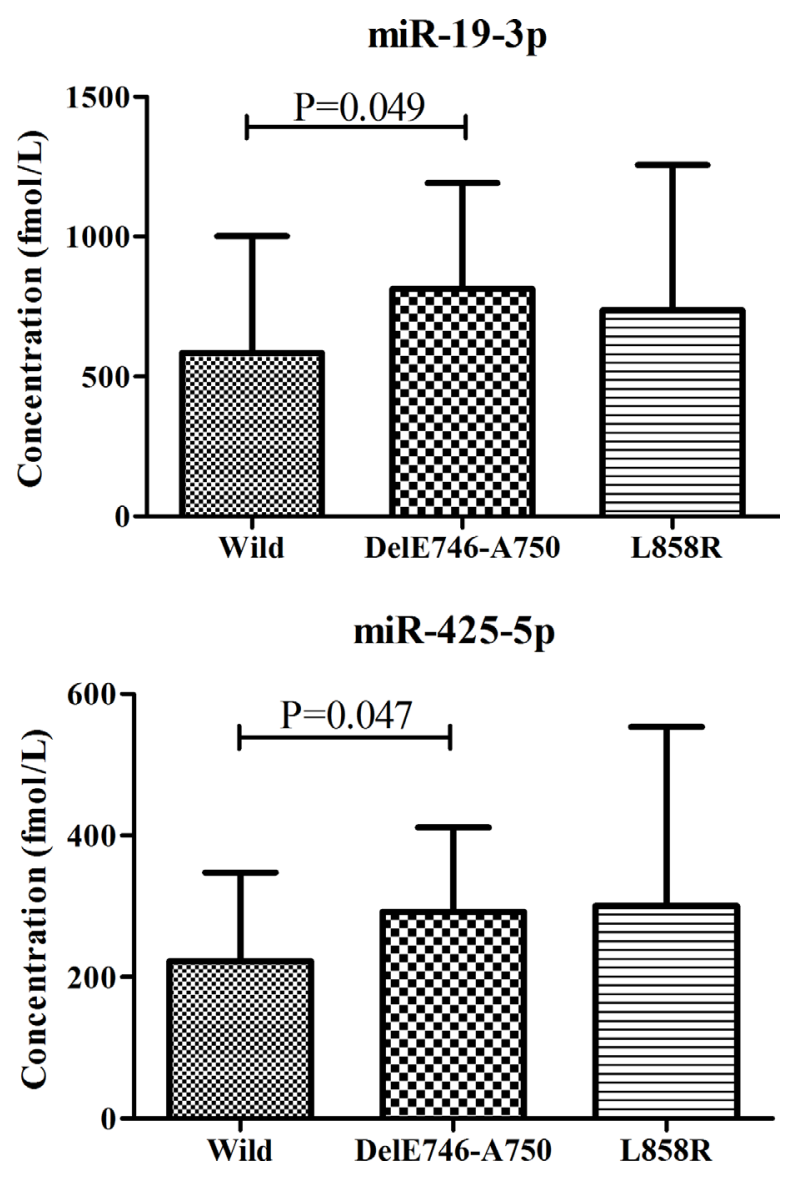

Figure 4: Different expression levels of miR-19-3p and miR-425-5p in peripheral plasma of LA patients according to EGFR status. LA: lung adenocarcinoma. 
regarding adjuvant treatment [51]. Nowadays, sequencing remains to be the gold standard for EGFR mutation analysis, but the positive rate is low. EGFR mutation could also be detected by PCR technology (amplification refractory mutation system, ARMS) which is simple and convenient but with some non-specific reactions and relatively high false positive rate [52]. To better understand the complicated mechanism and biology of lung cancer, many studies have explored the association of miRNAs and EGFR in lung cancer [53, 54]. Some studies also identified some circulating miRNAs as potential substitutes for EGFR mutation and biomarkers for monitoring EGFR-TKI treatment $[52,55]$. In our study, we found that plasma miR-19b-3p and miR-425-5p were significantly up-regulated in patients with DelE746-A750 mutation compared to those with wild type. Though without statistical significance, expression levels of the two plasma miRNAs were also higher in patients with L858R mutation compared to those with wild type. We thought that patients with high expression of plasma miR-
$19 b-3 p$ and miR-425-5p might be more sensitive to the EGFR-TKI treatment. However, due to the limited data, we did not explore the indicative role of the two miRNAs in monitoring the treatment effect of EGFR-TKI and the potentially prognostic value for LA patients in our study. Future study with more patients and comprehensive data is needed to validate our findings for the potential application of the miRNAs in the future clinical.

Derived from pulmonary circulation, arterial blood flows to systemic circulation and becomes venous plasma. Thus, we assumed that circulating miRNAs released from tumor cells might present higher expression levels in arterial blood than those in peripheral plasma. Our study revealed that miR-19b-3p, miR-21-5p, miR-221-3p, miR409-3p and miR-425-5p but not miR-584-5p in LA plasma might be released from LA cells since the consistent expression tendency between tissue and plasma samples. Interestingly, comparison of the miRNAs in peripheral and arterial plasma showed that the other five miRNAs except miR-584-5p were up-regulated in arterial plasma
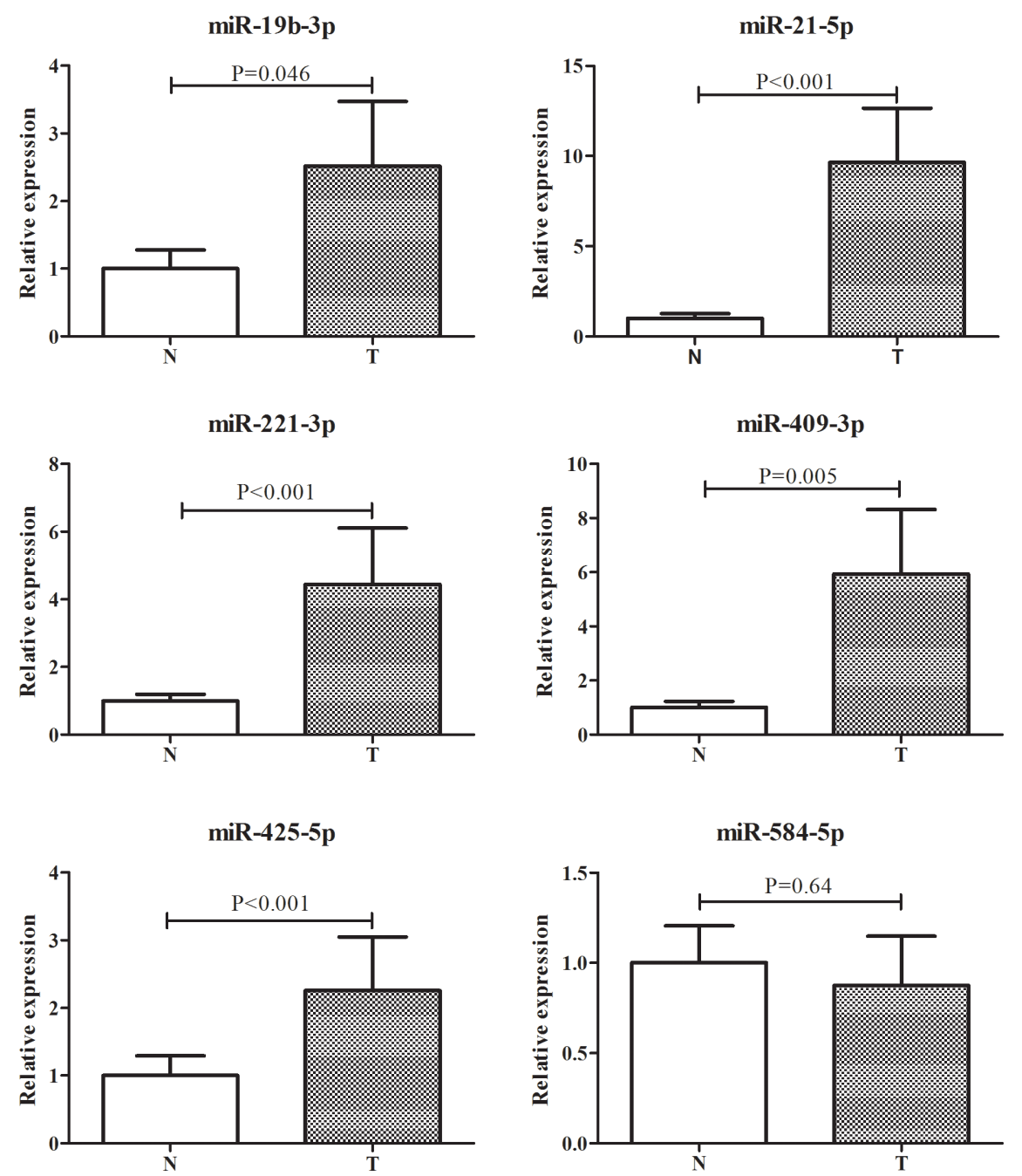

Figure 5: Expression of the six miRNAs in the tumor tissues of LA patients. $Y$ axis was presented as relative expression (normalized to $U 6 ; 2^{-\Delta \Delta \mathrm{Ct}}$ ). Error bar: standard error. LA: lung adenocarcinoma; N: normal tissues; T: tumor. 
in more than a half of the subjects. Although no result achieved statistical significance (miR-19-3p, miR-21-5p, miR-409-3p and miR-425-5p were borderline significant) potentially due to the small sample size, our findings could support the hypothesis in some extent.

Exosomes are small (40-100 nm) membrane vesicles secreted by most types of cell including cancer cell. Theses vesicles could protect the miRNAs, mRNAs and proteins from degradation by various enzymes outside and transfer messages among cells that make them the most important intercellular communicators [56]. Thus, the expression of the miRNAs identified in our study was also explored in exosomes to better understand the potential form of the miRNAs in peripheral plasma and utilize the potentially powerful diagnostic and therapeutic tool in the future. Our results showed that miR-19-3p, miR-21-5p and miR-221-3p were significantly upregulated in peripheral plasma exosomes from LA patients. Reportedly, high level of exosomal miR-21 could act as diagnostic and/or prognostic biomarker in some cancers $[57,58]$. The findings were consistent with our results. These three miRNAs might participate in the

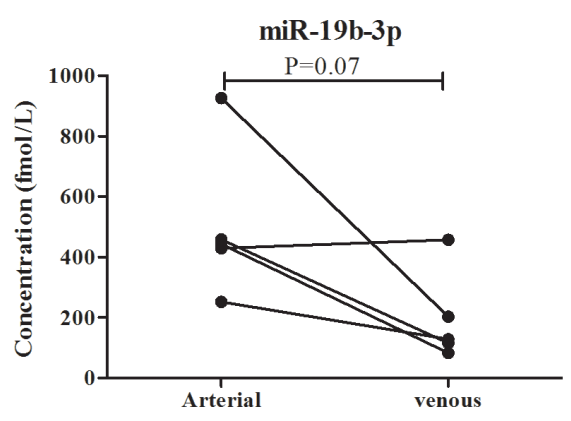

miR-221-3p

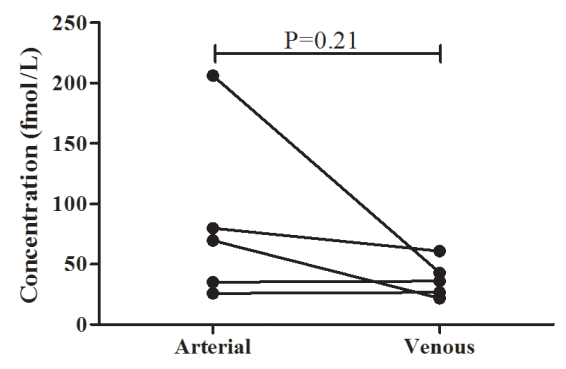

miR-425-5p

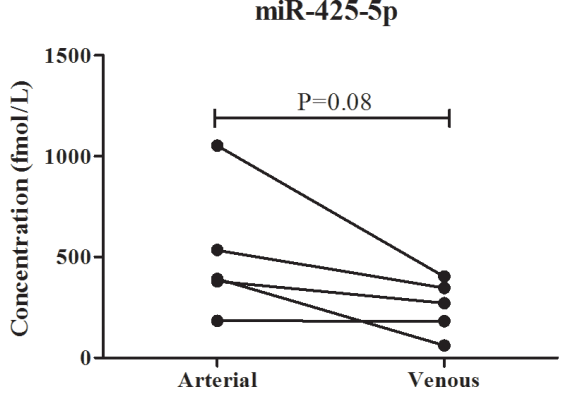

tumorgenesis and development of LA by exosomes and are worthy of further investigation. However, the other three miRNAs (miR-409-3p, miR-425-5p and miR-584-5p) did not show different expression between plasma exosomes from LA patients and HCs. It was reported that miRNAs were not only packaged into exosomes, they could also binding with proteins, such as the Argonaute 2 protein in the circulation [59]. Thus, we assumed that the majority of .miR-409-3p, miR-425-5p and miR-584-5p might bind to proteins in plasma of LA patients. The phenomena and the mechanism are warranted to be explored and validated in the future.

Some limitation of the study should be considered. First, Asian and Caucasian patients might have different molecular alterations, biological behaviors and clinical outcomes. However, we could not explore the role of the miRNAs in more patients with diverse populations. Future study with more cases with diverse populations might help to reveal the effect of population on the findings. Second, information about EGFR status was valid in 46 plasma samples. Only two types of mutation were included in our study. Meanwhile, the association of the identified
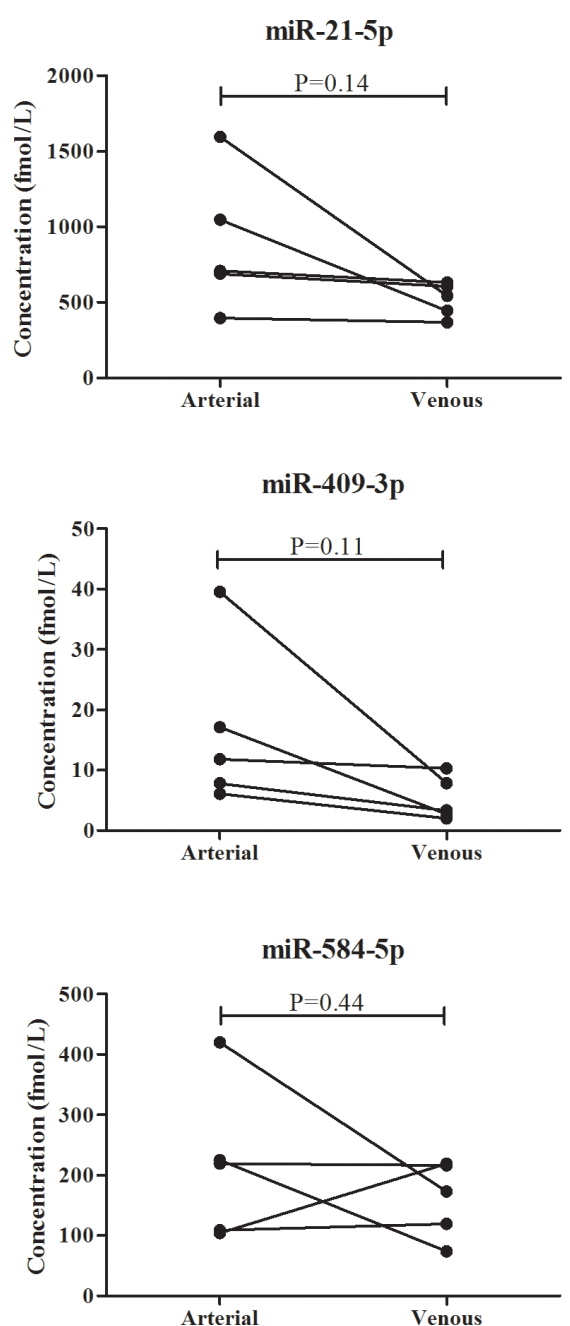

Figure 6: Comparison of the six miRNAs in 5 arterial plasma samples and matched peripheral plasma samples. 
miRNAs in tissue samples with EGFR was not explored. In the future, the relationship of the miRNAs and EGFR is needed to be explored widely. Third, only 5 pairs of arterial and peripheral venous blood samples were used in our study. Future studies with larger sample size are needed to validate the results of the miRNAs in arterial and peripheral plasma. In addition, our study focused on LA which was one subgroup of NSCLC. The diagnostic value and relationship of the miRNAs in other subtypes of NSCLC should also be explored in the future.

In conclusion, we identified a six-miRNA panel for LA detection. Our work will serve as the basis of the application of circulating miRNAs in clinical for the detection of LA in the future at least for Asian population to a certain extent. More effort should be taken to explore the roles and mechanisms of the miRNAs identified in our study in LA.

\section{MATERIALS AND METHODS}

\section{Study design, patients and samples}

All the subjects were recruited at First Affiliated Hospital of Nanjing Medical University between 2012 and 2014. All of the LA patients were histopathologically confirmed, and the tumor stage was determined according to the International Union Against Cancer's (UIAC) tumor-node-metastasis (TNM) system. Clinical characteristics for each patient were also recorded. And HCs enrolled in our study were healthy volunteers who conducted routine physical examination at First Affiliated Hospital of Nanjing Medical University

$5 \mathrm{ml}$ of blood sample from $\mathrm{HCs}$ and LA patients before initial treatment were collected with ethylenediaminetetraacetic acid (EDTA)-containing tubes (Becton,Dickinson and Company). The plasma was separated from blood within 12 hours after sample collection using a two-step protocol (350 RCF (reactive centrifugal force) for $10 \mathrm{~min}, 20,000 \mathrm{RCF}$ for $10 \mathrm{~min}$ (Beckman Coulter, USA)) and then stored at $-80^{\circ} \mathrm{C}$ for further processing. Tissue specimens were collected from surgery patients without preoperative chemoradiotherapy and kept in liquid nitrogen. The study was approved by Institutional Review Boards of the First Affiliated Hospital of Nanjing Medical University, and the written informed consent was obtained from each participant. The study was conducted according to the approved guidelines by the Hospital Ethics Committee.

Overall, a total of 141 LA patients and 124 HCs were enrolled in our study. The flow chart for the experiment design was shown in Figure 1. In the screening phase, Exiqon miRCURY-Ready-to-Use PCR-Human-panel-I+IIV1.M (Exiqon miRNA qPCR panel, Vedbaek, Denmark) was applied to identify differentially expressed miRNAs between 3 LA pool samples and 1 HC pool sample (30 peripheral plasma samples from LA patients and 10 from
HCs were randomly selected and per 10 samples were pooled as 1 pool sample). The process of arrays and analyses for the differential miRNA profiling in LA plasma was in accordance with the previous study [60]. Briefly, 25 ng RNA isolated from each pool sample was reverse transcribed and further subjected to the Exiqon panels to detect the 168 miRNAs with relatively high abundance in plasma/serum. The relative expression between LA patients and $\mathrm{HCs}$ was assessed by $2^{-\Delta \Delta \mathrm{Ct}}$ method, and $\Delta \mathrm{Ct}$ $=$ average $\mathrm{Ct}$ (assay) - average $\mathrm{Ct}$ (normalizer assays).

After that, to verify the reliability and reproducibility of the results from arrays, the dysregulated miRNAs were confirmed in the identical 40 samples by qRT-PCR using the $2^{-\Delta \Delta \mathrm{Ct}}$ method relative to cel-miR-39. In the training stage, the miRNAs discovered via the screening phase were determined in 42 LA samples and 32 HCs using qRT-PCR based absolute quantification methods. In the testing stage, the miRNAs identified in the training stage were further validated in plasma samples of 66 LA patients and $62 \mathrm{HCs}$. In the external validation stage, a total of 63 subjects including 33 cases and 30 controls were enrolled to further assess the diagnostic value of the identified miRNAs in LA.

An additional of nineteen pairs of LA tissue specimens and matched normal tissues were used to evaluate the expression level of identified miRNAs in tissue samples. Arterial blood samples were collected from $5 \mathrm{LA}$ patients and were identified to compare the difference of miRNAs between peripheral and arterial plasma. Exosomal miRNAs were further assessed in 18 LA patients and $14 \mathrm{HCs}$.

\section{Isolation of exosomes}

Exosomes from peripheral plasma were extracted using ExoQuick (System Biosciences, Mountain View, Calif) according to the manufacturer's protocol. Briefly, exosome pellets were precipitated from $100 \mu$ ExoQuick exosome precipitation solutions and $400 \mu \mathrm{l}$ plasma and then lysed in $200 \mu \mathrm{l}$ RNase-free water for RNA extraction.

\section{RNA extraction}

Total RNA from $200 \mu \mathrm{l}$ plasma or exosomes was isolated using the mirVana PARIS Kit (Ambion, Austin, TX, USA) in accordance with the protocol. After the addition of denaturing solution (Ambion, Austin, TX, USA), $5 \mu$ l synthetic C.elegans miR-39 (5 nM/L, RiboBio, Guangzhou, China) was spiked into each sample for normalization of variation between samples. According to the manufacturer's instructions, Trizol (Invitrogen, Carlsbad, CA, USA) was used to extract total RNA from tissue samples. Total RNA was lysed in $100 \mu$ RNasefree water and stored at $-80^{\circ} \mathrm{C}$ for further analysis. The concentration and purity of the total RNA was analyzed using the ultraviolet spectrophotometer. 


\section{Quantitative reverse transcription polymerase chain reaction}

Bulge-Loop ${ }^{\mathrm{TM}}$ miRNA qRT-PCR Primer Set (RiboBio, Guangzhou, China) including specific primers for reverse transcription (RT) and polymerase chain reaction (PCR) was used to amplify miRNAs. The expression levels of miRNAs were determined by evaluating the level of fluorescence emitted by SYBR Green $\left(\mathrm{SYBR}^{\circledR}\right.$ Premix Ex Taq ${ }^{\mathrm{TM}}$ II, TaKaRa, Dalian, China). RT and PCR were conducted as previously described [60-62]. Briefly, RT reactions were carried out at $42^{\circ} \mathrm{C}$ for $60 \mathrm{~min}$ followed by $70^{\circ} \mathrm{C}$ for $10 \mathrm{~min}$. The qRT-PCR was performed at $95^{\circ} \mathrm{C}$ for $20 \mathrm{sec}$, followed by 40 cycles of $95^{\circ} \mathrm{C}$ for $10 \mathrm{sec}, 60^{\circ} \mathrm{C}$ for $20 \mathrm{sec}$ and then $70^{\circ} \mathrm{C}$ for $10 \mathrm{sec}$ on $7900 \mathrm{HT}$ real-time PCR system (Applied Biosystems, Foster City, CA, USA). The specificity of PCR products was evaluated by the melting curve analysis. The expression level of plasma miRNAs evaluated in the training, testing and external validation stage was assessed as absolute concentration based on a standard curve constructed with the use of synthetic miRNAs (micrON miRNA mimic, RiboBio, Guangzhou, China) [61]. The expression of miRNAs from tissue samples and exosomes was determined using the $2^{-}$ ${ }^{\Delta \Delta \mathrm{Ct}}$ method relative to RNU6B (U6) and the combination of spiked in cel-miR-39 with miR-16.

\section{Statistical analysis}

The different expression levels of miRNAs were compared using paired $t$-test in paired arterial plasma and peripheral plasma samples and Mann-Whitney test in the other different groups. Clinical characteristics among different groups and their association with miRNA were evaluated with one-way ANOVA or $\chi^{2}$ test. The risk score of the identified miRNAs for LA was calculated $[60,63]$. The receiver operating characteristic (ROC) curves were applied to evaluate the optimal cutoff value for each miRNA. The score (S) for each miRNA was set as 1 if the expression level of the miRNA was greater than the cutoff value, otherwise as 0 . The risk score function (RSF) for subject i was: $\operatorname{RSF}_{i}=\sum_{j-1}^{k}\left(W_{j} * S_{i j}\right)$

Here, $\mathrm{S}_{\mathrm{ij}}$ is the risk score of miRNA $j$ on subject $i$ and weighted by the regression coefficient $\left(\mathrm{W}_{\mathrm{i}}\right)$ estimated from univariate logistic regression models for each miRNA. The area under the ROC curves (AUC) was used to investigate the diagnostic value of the miRNAs for LA.

All the statistical analyses were performed with the use of SPSS16.0 software (SPSS Inc., Chicago, IL, USA). A twosided $P$ value $<0.05$ was defined as statistical significance.

\section{ACKNOWLEDGMENTS AND FUNDING}

The authors are grateful to the fund support by the National Natural Science Foundation of China (Grant number: 81672400; 81272532; 81672788; 81302047) and
Jiangsu Province Clinical science and technology projects (Clinical Research Center, BL2012008).

\section{CONFLICTS OF INTEREST}

The authors declare no competing financial interests.

\section{REFERENCES}

1. Siegel R, Ma J, Zou Z and Jemal A. Cancer statistics, 2014. CA Cancer J Clin. 2014; 64:9-29.

2. Cataldo VD, Gibbons DL, Perez-Soler R, QuintasCardama A. Treatment of non-small-cell lung cancer with erlotinib or gefitinib. N Engl J Med. 2011; 364:947-955.

3. Cagle PT, Allen TC, Dacic S, Beasley MB, Borczuk AC, Chirieac LR, Laucirica R, Ro JY, Kerr KM. Revolution in lung cancer: new challenges for the surgical pathologist. Arch Pathol Lab Med. 2011; 135:110-116.

4. Chen Z, Fillmore CM, Hammerman PS, Kim CF, Wong KK. Non-small-cell lung cancers: a heterogeneous set of diseases. Nat Rev Cancer. 2014; 14:535-546.

5. Rosell R, Bivona TG, Karachaliou N. Genetics and biomarkers in personalisation of lung cancer treatment. Lancet. 2013; 382:720-731.

6. Goldstraw P, Ball D, Jett JR, Le Chevalier T, Lim E, Nicholson AG, Shepherd FA. Non-small-cell lung cancer. Lancet. 2011; 378:1727-1740.

7. Li Q, Liu M, Ma F, Luo Y, Cai R, Wang L, Xu N, Xu B. Circulating miR-19a and miR-205 in serum may predict the sensitivity of luminal A subtype of breast cancer patients to neoadjuvant chemotherapy with epirubicin plus paclitaxel. PLoS One. 2014; 9:e104870.

8. Aberle DR, Adams AM, Berg CD, Black WC, Clapp JD, Fagerstrom RM, Gareen IF, Gatsonis C, Marcus PM, Sicks JD. Reduced lung-cancer mortality with low-dose computed tomographic screening. N Engl J Med. 2011; 365:395-4 09.

9. Okamura K, Takayama K, Izumi M, Harada T, Furuyama K, Nakanishi Y. Diagnostic value of CEA and CYFRA 21-1 tumor markers in primary lung cancer. Lung Cancer. 2013; 80:45-49.

10. Tarro G, Perna A, Esposito C. Early diagnosis of lung cancer by detection of tumor liberated protein. J Cell Physiol. 2005; 203:1-5.

11. Lee RC, Feinbaum RL, Ambros V. The C. elegans heterochronic gene lin-4 encodes small RNAs with antisense complementarity to lin-14. Cell. 1993; 75:843-854.

12. Lewis BP, Burge CB, Bartel DP. Conserved seed pairing, often flanked by adenosines, indicates that thousands of human genes are microRNA targets. Cell. 2005; 120:15-20.

13. Schwarzenbach H, Nishida N, Calin GA, Pantel K. Clinical relevance of circulating cell-free microRNAs in cancer. Nat Rev Clin Oncol. 2014; 11:145-156.

14. Zandberga E, Kozirovskis V, Abols A, Andrejeva D, Purkalne G, Line A. Cell-free microRNAs as diagnostic, 
prognostic, and predictive biomarkers for lung cancer. Genes Chromosomes Cancer. 2013; 52:356-369.

15. Langer CJ, Besse B, Gualberto A, Brambilla E, Soria JC. The evolving role of histology in the management of advanced non-small-cell lung cancer. J Clin Oncol. 2010; 28:5311-5320.

16. Wilkerson MD, Yin X, Walter V, Zhao N, Cabanski CR, Hayward MC, Miller CR, Socinski MA, Parsons AM, Thorne LB, Haithcock BE, Veeramachaneni NK, Funkhouser WK, et al. Differential pathogenesis of lung adenocarcinoma subtypes involving sequence mutations, copy number, chromosomal instability, and methylation. PLoS One. 2012; 7:e36530.

17. Comprehensive molecular profiling of lung adenocarcinoma. Nature. 2014; 511:543-550.

18. McDonald JS, Milosevic D, Reddi HV, Grebe SK, Algeciras-Schimnich A. Analysis of circulating microRNA: preanalytical and analytical challenges. Clin Chem. 2011; 57:833-840.

19. Sellars MJ, Vuocolo T, Leeton LA, Coman GJ, Degnan BM, Preston NP. Real-time RT-PCR quantification of Kuruma shrimp transcripts: a comparison of relative and absolute quantification procedures. J Biotechnol. 2007; 129:391-399.

20. Kroh EM, Parkin RK, Mitchell PS, Tewari M. Analysis of circulating microRNA biomarkers in plasma and serum using quantitative reverse transcription-PCR (qRT-PCR). Methods. 2010; 50:298-301.

21. Hu J, Wang Z, Liao BY, Yu L, Gao X, Lu S, Wang S, Dai Z, Zhang X, Chen Q, Qiu SJ, Wu Y, Zhu H, et al. Human miR-1228 as a stable endogenous control for the quantification of circulating microRNAs in cancer patients. Int J Cancer. 2014; 135:1187-1194.

22. Jensen SG, Lamy P, Rasmussen MH, Ostenfeld MS, Dyrskjot L, Orntoft TF, Andersen CL. Evaluation of two commercial global miRNA expression profiling platforms for detection of less abundant miRNAs. BMC Genomics. 2011; 12:435.

23. Li J, Yang S, Yan W, Yang J, Qin YJ, Lin XL, Xie RY, Wang SC, Jin W, Gao F, Shi JW, Zhao WT, Jia JS, et al. MicroRNA-19 triggers epithelial-mesenchymal transition of lung cancer cells accompanied by growth inhibition. Lab Invest. 2015.

24. Wu C, Cao Y, He Z, He J, Hu C, Duan H, Jiang J. Serum levels of miR-19b and miR-146a as prognostic biomarkers for non-small cell lung cancer. Tohoku J Exp Med. 2014; 232:85-95.

25. Baer C, Claus R, Plass C. Genome-wide epigenetic regulation of miRNAs in cancer. Cancer Res. 2013; 73:473-477.

26. Nair VS, Maeda LS, Ioannidis JP. Clinical outcome prediction by microRNAs in human cancer: a systematic review. J Natl Cancer Inst. 2012; 104:528-540.

27. Yu L, Todd NW, Xing L, Xie Y, Zhang H, Liu Z, Fang H, Zhang J, Katz RL, Jiang F. Early detection of lung adenocarcinoma in sputum by a panel of microRNA markers. Int J Cancer. 2010; 127:2870-2878.
28. Vosa U, Vooder T, Kolde R, Vilo J, Metspalu A, Annilo T. Meta-analysis of microRNA expression in lung cancer. Int J Cancer. 2013; 132:2884-2893.

29. Ferracin M, Lupini L, Salamon I, Saccenti E, Zanzi MV, Rocchi A, Da Ros L, Zagatti B, Musa G, Bassi C, Mangolini A, Cavallesco G, Frassoldati A, et al. Absolute quantification of cell-free microRNAs in cancer patients. Oncotarget. 2015; 6:14545-14555. doi: 10.18632/ oncotarget.3859.

30. Saito M, Schetter AJ, Mollerup S, Kohno T, Skaug V, Bowman ED, Mathe EA, Takenoshita S, Yokota J, Haugen A, Harris CC. The association of microRNA expression with prognosis and progression in early-stage, non-small cell lung adenocarcinoma: a retrospective analysis of three cohorts. Clin Cancer Res. 2011; 17:1875-1882.

31. Gao W, Lu X, Liu L, Xu J, Feng D, Shu Y. MiRNA-21: a biomarker predictive for platinum-based adjuvant chemotherapy response in patients with non-small cell lung cancer. Cancer Biol Ther. 2012; 13:330-340.

32. Geng Q, Fan T, Zhang B, Wang W, Xu Y, Hu H. Five microRNAs in plasma as novel biomarkers for screening of early-stage non-small cell lung cancer. Respir Res. 2014; 15:149.

33. Yamashita R, Sato M, Kakumu T, Hase T, Yogo N, Maruyama E, Sekido Y, Kondo M, Hasegawa Y. Growth inhibitory effects of miR-221 and miR-222 in non-small cell lung cancer cells. Cancer Med. 2015; 4:551-564.

34. Garofalo M, Di Leva G, Romano G, Nuovo G, Suh SS, Ngankeu A, Taccioli C, Pichiorri F, Alder H, Secchiero P, Gasparini P, Gonelli A, Costinean S, et al. miR-221\&222 regulate TRAIL resistance and enhance tumorigenicity through PTEN and TIMP3 downregulation. Cancer Cell. 2009; 16:498-509.

35. Garofalo M, Quintavalle C, Di Leva G, Zanca C, Romano G, Taccioli C, Liu CG, Croce CM, Condorelli G. MicroRNA signatures of TRAIL resistance in human non-small cell lung cancer. Oncogene. 2008; 27:3845-3855.

36. Heegaard NH, Schetter AJ, Welsh JA, Yoneda M, Bowman ED, Harris CC. Circulating micro-RNA expression profiles in early stage nonsmall cell lung cancer. Int $\mathrm{J}$ Cancer. 2012; 130:1378-1386.

37. Wang J, Li Z, Ge Q, Wu W, Zhu Q, Luo J, Chen L. Characterization of microRNA transcriptome in tumor, adjacent, and normal tissues of lung squamous cell carcinoma. J Thorac Cardiovasc Surg. 2015; 149:1404-1414 e1404.

38. Josson S, Gururajan M, Sung SY, Hu P, Shao C, Zhau HE, Liu C, Lichterman J, Duan P, Li Q, Rogatko A, Posadas EM, Haga CL, et al. Stromal fibroblast-derived miR-409 promotes epithelial-to-mesenchymal transition and prostate tumorigenesis. Oncogene. 2015; 34:2690-2699.

39. Zheng B, Liang L, Huang S, Zha R, Liu L, Jia D, Tian Q, Wang Q, Wang C, Long Z, Zhou Y, Cao X, Du C, et al. MicroRNA-409 suppresses tumour cell invasion and metastasis by directly targeting radixin in gastric cancers. Oncogene. 2012; 31:4509-4516. 
40. Wang XP, Deng XL, Li LY. MicroRNA-584 functions as a tumor suppressor and targets PTTG1IP in glioma. Int J Clin Exp Pathol. 2014; 7:8573-8582.

41. Gaedcke J, Grade M, Camps J, Sokilde R, Kaczkowski B, Schetter AJ, Difilippantonio MJ, Harris CC, Ghadimi BM, Moller S, Beissbarth T, Ried T, Litman T. The rectal cancer microRNAome--microRNA expression in rectal cancer and matched normal mucosa. Clin Cancer Res. 2012; 18:4919-4930.

42. Zhang J, Song Y, Zhang C, Zhi X, Fu H, Ma Y, Chen Y, Pan F, Wang K, Ni J, Jin W, He X, Su H, et al. Circulating MiR16-5p and MiR-19b-3p as Two Novel Potential Biomarkers to Indicate Progression of Gastric Cancer. Theranostics. 2015; 5:733-745.

43. Huang JT, Liu SM, Ma H, Yang Y, Zhang X, Sun H, Xu J, Wang J. Systematic Review and Meta-Analysis: Circulating miRNAs for Diagnosis of Hepatocellular Carcinoma. J Cell Physiol. 2015.

44. Wu J, Li G, Wang Z, Yao Y, Chen R, Pu X, Wang J. Circulating MicroRNA-21 Is a Potential Diagnostic Biomarker in Gastric Cancer. Dis Markers. 2015; 2015:435656.

45. Cai H, Yuan Y, Hao YF, Guo TK, Wei X, Zhang YM. Plasma microRNAs serve as novel potential biomarkers for early detection of gastric cancer. Med Oncol. 2013; 30:452.

46. Pu XX, Huang GL, Guo HQ, Guo CC, Li H, Ye S, Ling S, Jiang L, Tian Y, Lin TY. Circulating miR-221 directly amplified from plasma is a potential diagnostic and prognostic marker of colorectal cancer and is correlated with p53 expression. J Gastroenterol Hepatol. 2010; 25:1674-1680.

47. Kanemaru H, Fukushima S, Yamashita J, Honda N, Oyama R, Kakimoto A, Masuguchi S, Ishihara T, Inoue Y, Jinnin M, Ihn H. The circulating microRNA-221 level in patients with malignant melanoma as a new tumor marker. J Dermatol Sci. 2011; 61:187-193.

48. Yilmaz SS, Guzel E, Karatas OF, Yilmaz M, Creighton CJ, Ozen M. MiR-221 as a pre- and postoperative plasma biomarker for larynx cancer patients. Laryngoscope. 2015.

49. Kawaguchi $\mathrm{T}$, Komatsu S, Ichikawa D, Morimura R, Tsujiura M, Konishi H, Takeshita H, Nagata H, Arita T, Hirajima S, Shiozaki A, Ikoma H, Okamoto K, et al. Clinical impact of circulating miR-221 in plasma of patients with pancreatic cancer. Br J Cancer. 2013; 108:361-369.

50. Jorge SE, Kobayashi SS, Costa DB. Epidermal growth factor receptor (EGFR) mutations in lung cancer: preclinical and clinical data. Braz J Med Biol Res. 2014; 47:929-939.

51. Li T, Kung HJ, Mack PC, Gandara DR. Genotyping and genomic profiling of non-small-cell lung cancer: implications for current and future therapies. J Clin Oncol. 2013; 31:1039-1049.

52. Zhao Q, Cao J, Wu YC, Liu X, Han J, Huang XC, Jiang LH, Hou XX, Mao WM, Ling ZQ. Circulating miRNAs is a potential marker for gefitinib sensitivity and correlation with EGFR mutational status in human lung cancers. Am J Cancer Res. 2015; 5:1692-1705.
53. Chan LW, Wang FF, Cho WC. Genomic sequence analysis of EGFR regulation by microRNAs in lung cancer. Curr Top Med Chem. 2012; 12:920-926.

54. Han F, He J, Li F, Yang J, Wei J, Cho WC, Liu X. Emerging Roles of MicroRNAs in EGFR-Targeted Therapies for Lung Cancer. Biomed Res Int. 2015; 2015:672759.

55. Sun W, Yuan X, Tian Y, Wu H, Xu H, Hu G, Wu K. Non-invasive approaches to monitor EGFR-TKI treatment in non-small-cell lung cancer. J Hematol Oncol. 2015; 8:95.

56. Mathivanan S, Ji H, Simpson RJ. Exosomes: extracellular organelles important in intercellular communication. J Proteomics. 2010; 73:1907-1920.

57. Tanaka Y, Kamohara H, Kinoshita K, Kurashige J, Ishimoto T, Iwatsuki M, Watanabe M, Baba H. Clinical impact of serum exosomal microRNA-21 as a clinical biomarker in human esophageal squamous cell carcinoma. Cancer. 2013; 119:1159-1167.

58. Wang J, Zhou Y, Lu J, Sun Y, Xiao H, Liu M, Tian L. Combined detection of serum exosomal miR-21 and HOTAIR as diagnostic and prognostic biomarkers for laryngeal squamous cell carcinoma. Med Oncol. 2014; 31:148.

59. Arroyo JD, Chevillet JR, Kroh EM, Ruf IK, Pritchard CC, Gibson DF, Mitchell PS, Bennett CF, Pogosova-Agadjanyan EL, Stirewalt DL, Tait JF, Tewari M. Argonaute2 complexes carry a population of circulating microRNAs independent of vesicles in human plasma. Proc Natl Acad Sci USA. 2011; 108:5003-5008.

60. Zhou X, Zhu W, Li H, Wen W, Cheng W, Wang F, Wu Y, Qi L, Fan Y, Chen Y, Ding Y, Xu J, Qian J, et al. Diagnostic value of a plasma microRNA signature in gastric cancer: a microRNA expression analysis. Sci Rep. 2015; 5:11251.

61. Zhao DS, Chen Y, Jiang H, Lu JP, Zhang G, Geng J, Zhang Q, Shen JH, Zhou X, Zhu W, Shan QJ. Serum miR210 and miR-30a expressions tend to revert to fetal levels in Chinese adult patients with chronic heart failure. Cardiovasc Pathol. 2013; 22:444-450.

62. Qiu T, Zhou X, Wang J, Du Y, Xu J, Huang Z, Zhu W, Shu Y, Liu P. MiR-145, miR-133a and miR-133b inhibit proliferation, migration, invasion and cell cycle progression via targeting transcription factor $\mathrm{Sp} 1$ in gastric cancer. FEBS Lett. 2014; 588:1168-1177.

63. Zhu C, Ren C, Han J, Ding Y, Du J, Dai N, Dai J, Ma H, $\mathrm{Hu}$ Z, Shen H, Xu Y, Jin G. A five-microRNA panel in plasma was identified as potential biomarker for early detection of gastric cancer. Br J Cancer. 2014; 110:2291-2299. 